\section{(2) OPEN ACCESS}

\title{
Miliary tuberculosis in a paediatric patient with psoriasis
}

\author{
Jacob Kilgore, ${ }_{1}^{1}$ Jonathon Pelletier, ${ }^{2}$ Bradford Becken, ${ }^{1}$ Stephen Kenny, ${ }^{3}$ Samrat Das, ${ }^{4}$ \\ Lisa Parnell ${ }^{4}$
}

${ }^{1}$ Division of Pediatric Infectious Diseases, Duke University Hospital, Durham, North Carolina, USA

2Division of Pediatric of Critical Care Medicine, UPMC Pittsburgh, Pennsylvania, USA ${ }^{3}$ Department of Pediatrics, King Edward VII Memorial Hospital, Paget, Bermuda

${ }^{4}$ Department of Pediatrics, Duke University Hopistal, Durham, North Carolina, USA

Correspondence to

Dr Bradford Becken; bbecken03@gmail.com

Accepted 23 January 2021

Check for updates

(c) BMJ Publishing Group Limited 2021. Re-use permitted under CC BY-NC. No commercial re-use. See rights and permissions. Published by BMJ.

To cite: Kilgore J, Pelletier J, Becken $B$, et al. BMJ Case Rep 2021;14:e237580. doi:10.1136/bcr-2020237580

\section{SUMMARY}

We present a 16-year-old girl with a history of wellcontrolled psoriasis, on immunosuppression, who sought evaluation in the emergency department for 4 months of fever, cough and unintentional weight loss. The patient had seen multiple providers who had diagnosed her with community-acquired pneumonia, but she was unimproved after oral antibiotic therapy. On presentation, she was noted to be febrile, tachycardic and chronically ill-appearing. Her chest X-ray showed diffuse opacities and a right upper lobe cavitary lesion concerning for tuberculosis. A subsequent chest CT revealed miliary pulmonary nodules in addition to the cavitary lesion. The patient underwent subsequent brain MRI, which revealed multifocal ring-enhancing nodules consistent with parenchymal involvement. The patient was diagnosed with miliary tuberculosis and improved on quadruple therapy. Though rates of tuberculosis are increasing, rates remain low in children, though special consideration should be given to children who are immunosuppressed.

\section{BACKGROUND}

Tuberculosis remains relatively rare in children compared with adults but select paediatric patientpopulations remain at an increased risk, one such population being immunosuppressed children. Given the growing availability and application of immunomodulatory medications for both autoimmune and transplant indications, there exists a growing population of children who are at an increased risk for the acquisition and deleterious effects of tuberculosis.

\section{CASE PRESENTATION}

A 16-year-old girl with a history of psoriasis presented to the emergency department for fever, cough and unintentional weight loss. Her symptoms began 4 months prior to presentation with intermittent, non-productive daytime cough associated with pleuritic chest pain. She describes daily tactile fevers and chills. She has also had intermittent diffuse, 'crampy' abdominal pain with associated non-bloody, non-bilious emesis occurring approximately three times per week. Additionally, she described nausea and anorexia, resulting in a $15 \mathrm{~kg}$ weight loss over the past 4 months. She denied upper respiratory symptoms, sore throat, dysphagia, haematemesis, haemoptysis, cyanosis, diarrhoea, melena, haematochezia, dysuria, haematuria or rashes. She denied syncope, presyncope or seizures. She reported her last menstrual period as 3 months prior and denied any current vaginal symptoms. She denied recent travel, known sick contacts, sexual activity or illicit and recreational drug use. She was born in Ethiopia, moved to the USA relatively soon after birth and last travelled there 9 years ago. She had no contact with recent international travellers or prisoners.

She reported that during the past 4 months, she had been seen at an urgent care facility multiple times and treated with three different oral antibiotics for pneumonia; she cannot recall the names of these prescriptions. She has also been seen in an outpatient gastroenterology clinic and had an oesophagogastroduodenoscopy that demonstrated oesophageal candidiasis, for which she was treated with fluconazole. She was previously healthy with the exception of psoriasis. Regarding her psoriasis, she reported that she previously had diffuse skin involvement, but has never had arthritis. She was initially treated with adalimumab, a monoclonal antibody against tumour necrosis factor (TNF)- $\alpha$, but was changed to methotrexate after being diagnosed with pneumonia. Her psoriatic symptoms are currently well controlled. She presented to the emergency department due to lack of improvement in systemic symptoms after the above management and a new sensation of a racing heartbeat.

In the emergency department, her initial vital signs were: temperature $39.2^{\circ} \mathrm{C}$, heart rate of 140 beats/min, respiratory rate of 18 breaths/min, blood pressure of $115 / 61 \mathrm{~mm} \mathrm{Hg}$, oxygen saturation of $94 \%$ on room air and weight $54.6 \mathrm{~kg}$. On examination, she was tired and chronically ill-appearing, but non-toxic. Her physical examination was remarkable for tachycardia without murmur, bounding radial pulses and mild respiratory distress with asymmetrically (right $>$ left) diminished basilar aeration. She does not have lymphadenopathy, hepatosplenomegaly, ascites or peripheral oedema. The remainder of her examination was normal. Laboratory studies were remarkable for normocytic anaemia, lymphopenia, hypoalbuminemia, elevated prothrombin time and elevated inflammatory markers. Electrolytes, transaminases, thyroid profile and urine pregnancy test were normal. HIV antibody and viral load were negative. Review of records shows that her outside chest X-ray was remarkable for rounded opacities scattered throughout both lungs and a right upper lobe cavitary lesion. A chest CT was obtained, which reveals a cavitary right upper lobe mass (figure 1). 


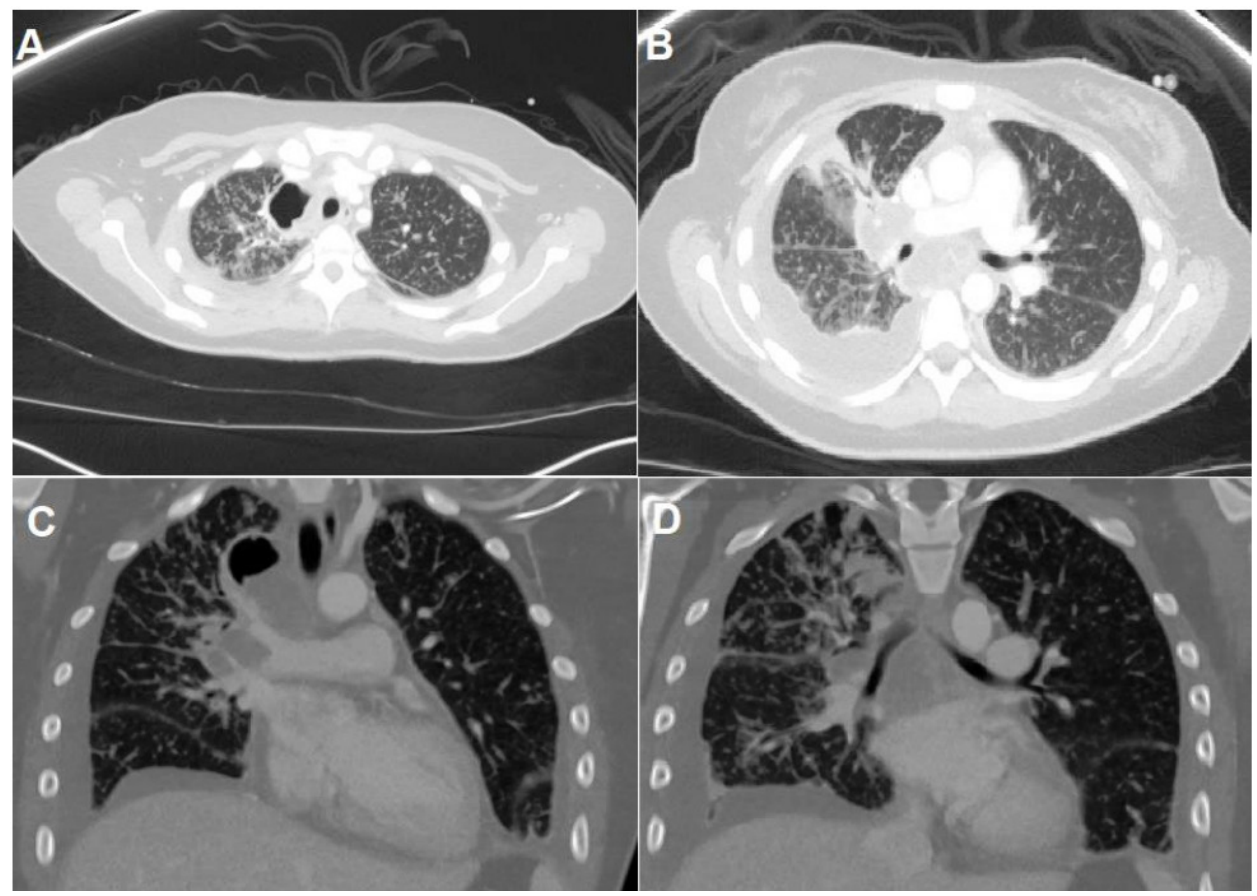

Figure 1 Contrast-enhanced $\mathrm{CT}$ of the chest shows a $3 \mathrm{~cm}$ round right upper cavitary lesion as well as prominent miliary nodules within the right upper lobe on axillary section (panel A). Further sections show multiple large necrotic mediastinal lymph nodes (panel B). coronal reformatting allows visualisation of the cavitary lesion (panel C) and miliary nodules (panel D) in plane with mediastinal lymphadenopathy.

\section{DIFFERENTIAL DIAGNOSIS}

The patient's chest CT revealed a cavitary right upper lobe mass, miliary pulmonary nodules and necrotic mediastinal lymphadenopathy. The radiographical differential of cavitary lesions and miliary nodules included congenital pulmonary airway malformations, lung abscesses, septic emboli, tuberculosis, histoplasmosis, blastomycosis, sarcoidosis and malignancies. ${ }^{12}$ However, the combination of findings in the setting of immunosuppression were highly suspicious for Mycobacterium tuberculosis.

\section{INVESTIGATIONS}

Due to the strong suspicion for M. tuberculosis after her chest imaging, sputum PCR and cultures were obtained and were positive. She was diagnosed with miliary tuberculosis. Given her immunosuppression, she underwent evaluation for haematogenous seeding of M. tuberculosis. Brain MRI revealed multifocal ring-enhancing nodules consistent with parenchymal central nervous system (CNS) involvement (figure 2). MRI did not show meningeal enhancement, and a subsequent lumbar puncture revealed unremarkable cerebrospinal fluid parameters.

\section{TREATMENT AND CLINICAL COURSE}

The patient was admitted to the hospital and maintained under negative pressure isolation. She was started on isoniazid, pyrazinamide, rifampin and moxifloxacin. Resistance testing revealed a pan-sensitive isolate. The local health department was notified, and her family members were tested for tuberculosis. She was discharged with a plan for extended direct observed therapy.

\section{OUTCOME AND FOLLOW-UP}

The patient remained on medication for 1 year following diagnosis and did not endorse any side effects for her antimycobacterial therapy. Throughout her time on therapy, as well as the subsequent 12 months, she has remained free of any flares of her psoriasis. Her psoriasis is now being managed with topical medications only. The patient was enrolling in college in the Autumn of 2020. Owing to her hospitalisation for tuberculosis and interest in her presentation, she was considering pursuing a course of study involving public health and/or medicine.

\section{DISCUSSION}

Cases of disseminated or military tuberculosis, a disease caused by the acid-fast bacillus M. tuberculosis, secondary to TNF- $\alpha$ inhibitors are becoming more commonplace, though they remain rare in paediatric patients. ${ }^{4-8}$ However, the increasing use of immunomodulatory medications such as TNF- $\alpha$ inhibitors is increasing the risk of presentation. Testing for latent tuberculosis remains imperative prior to initiating immunomodulatory therapy, but it is unable to prevent all infections. Well-established treatment guidelines exist, but not all antituberculosis drugs have been well-studied in children. ${ }^{9}$ Furthermore, as was the case here, treatment regimens must be altered when there is CNS involvement. ${ }^{10}$

According to the Centers for Disease Control and Prevention, one-quarter of the world's population is infected with $M$. tuberculosis. ${ }^{11}$ The incidence in the USA has fallen dramatically from $52.6 / 100000$ persons in 1953 to $2.9 / 100000$ persons in 2016. ${ }^{11}$ Children constitute approximately $14 \%$ of new cases. ${ }^{11}$ The falling rates of tuberculosis, and the low incidence in children, likely played a role in the patient's delayed diagnosis. Risk factors for infection include Asian descent, recent immigration to the USA, diabetes, immunodeficiency, homelessness and incarceration. ${ }^{11}$ In the majority of infected persons, infection is followed by latent disease with no clinical symptoms. ${ }^{12}$ In children under 10 years, age of exposure is inversely correlated with the probability of developing clinical disease; nearly $50 \%$ of exposed infants under 1 year become symptomatic. ${ }^{12} 13$ There is a smaller peak of infection in adolescence, with the vast majority of these patients developing pulmonary disease. ${ }^{12} 13$ 


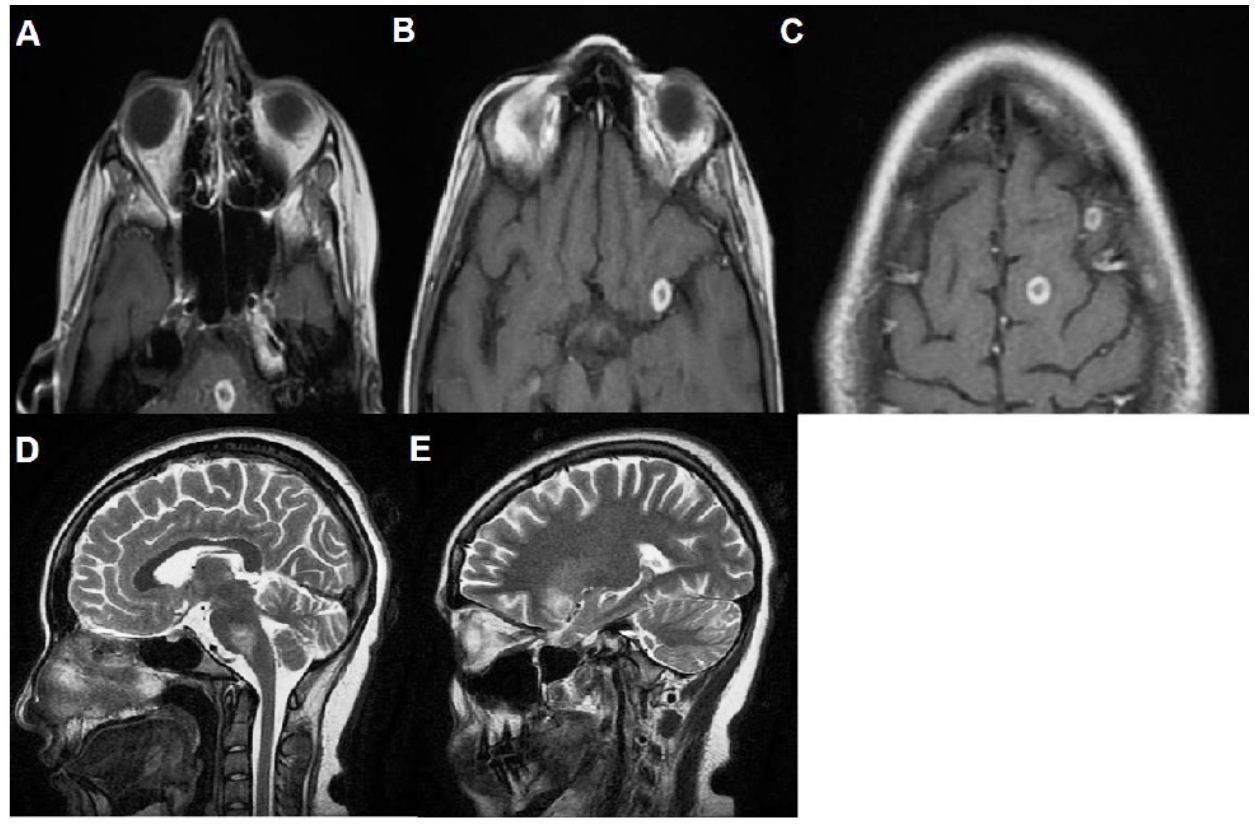

Figure 2 Contrast-enhanced MRI of the brain shows multifocal ring-enhancing lesions in the pons and cerebellum (panel A and D), and throughout the supratentorial brain (panel B, C and E), consistent with caseating tuberculous granulomas.

The spectrum of clinical disease caused by M. tuberculosis includes asymptomatic infection (latent tuberculosis), respiratory symptoms (pulmonary tuberculosis), and multisystem disease with or without pulmonary involvement (miliary tuberculosis). ${ }^{14} 15$ The age at acquisition strongly influences the form of disease that develops in the child, with one study looking at children ages 0-18 years showing that more than 60\% present with disseminated disease. ${ }^{14-16}$ For children with pulmonary disease, the hallmark clinical symptoms in all age groups are chronic cough, fever and weight loss. ${ }^{15}{ }^{17}$ Cough is rarely productive in infants or children, but may be in adolescents. ${ }^{15}$ Infants more typically present with examination features compatible with pneumonia, whereas adolescents typically lack physical findings. ${ }^{15}$ Chest X-ray may be diagnostic, though CT has higher sensitivity. ${ }^{3}$ Thick-walled cavitary lesions with ipsilateral hilar or paratracheal lymphadenopathy, miliary nodules and pleural effusions are all suggestive of pulmonary tuberculosis. ${ }^{3}$

Infants are much more likely than immunocompetent older children to develop extrapulmonary tuberculosis. ${ }^{12} 1518$ Moreover, paediatric patients have an increased risk of CNS involvement. ${ }^{8}$ While no large studies have been conducted in children, retrospective reviews in adults have not been able to establish a clear link between CNS involvement and outcome. ${ }^{19}$ However, in two studies of adult patients maintained on antiTNF- $\alpha$, such as adalimumab, more than half of those who contracted tuberculosis developed extrapulmonary manifestations. ${ }^{20} \quad 21$ Extrapulmonary tuberculosis presents with variable symptoms depending on the affected organ system. The most common sites of extrapulmonary tuberculosis are lymph nodes, CNS, pleura, and the skeletal system. ${ }^{15} 18 \mathrm{CNS}$ tuberculosis is seen in approximately $50 \%$ of extrapulmonary cases and most often manifests as basilar meningitis; parenchymal tuberculomas are seen less frequently. ${ }^{15} 2223$ CT or MRI can be used to screen for CNS tuberculosis. ${ }^{18}$ Obstructive hydrocephalus is the most common imaging finding, followed by basilar meningeal enhancement and infarction of the basal ganglia, thalami or anterior limbs of the internal capsules. ${ }^{18}$ Disseminated ring-enhancing caseating granulomas may be seen, and are often infratentorial in children. ${ }^{18}$ Abdominal tuberculosis is less common, but may include hepatic, splenic, renal, gastrointestinal, most commonly ileocecal or adrenal involvement. ${ }^{24}$

Diagnosis of tuberculosis is definitively established by the combination of a suspected exposure, clinical and imaging features consistent with disease, and isolation of M. tuberculosis in bacterial culture from sputum, bronchoscopy or biopsy specimen. $^{12} 1425$ Unfortunately, growth of M. tuberculosis in culture medium typically requires $1-2$ weeks. ${ }^{25}$ Thus, when clinical suspicion of pulmonary tuberculosis is moderate or high, the Infectious Diseases Society of America (IDSA) recommends sending three sputum samples for acid-fast staining and nucleic acid amplification testing. ${ }^{25}$ The use of the two tests in combination on multiple samples increases sensitivity and specificity, and provides results in hours. ${ }^{25}$ However, false-negative results occur, and these tests should not be used to rule out the diagnosis. ${ }^{25}$

Treatment of tuberculosis depends on clinical manifestations. Patients with latent tuberculosis are usually treated with 9 months of isoniazid. ${ }^{26}$ For patients with pulmonary tuberculosis, the IDSA recommends 8 weeks of isoniazid, pyrazinamide, rifampin and ethambutol, followed by 18 weeks of isoniazid and rifampin. ${ }^{9}$ In children with CNS disease, the American Academy of Pediatrics recommends isoniazid, pyrazinamide, rifampin, and ethionamide or an aminoglycoside for 8 weeks followed by

\section{Learning points}

Children account for approximately $14 \%$ of all diagnosis of tuberculosis. $^{11}$

- The spectrum of presentation of children with tuberculosis range from asymptomatic to multisystem involvement, and is strongly influenced by age. ${ }^{814-16}$

- Central nervous system involvement occurs in $50 \%$ of patients with extrapulmonary involvement. ${ }^{8152223}$

- The use of tumour necrosis factor- $\alpha$ inhibitors increase the risk of acquiring tuberculosis. ${ }^{4-6}$ 
$28-40$ weeks of isoniazid and rifampin. ${ }^{9}$ In adults, moxifloxacin may be used in place of ethionamide. ${ }^{27-29}$ Molecular testing for rifampin sensitivity is recommended in patients with risk factors for resistance. ${ }^{25}$ In tuberculous meningitis, the IDSA recommends the addition of corticosteroids tapered over $6-8$ weeks. ${ }^{9}$ Corticosteroids should also be considered for evidence of immune reconstitution inflammatory syndrome. ${ }^{30}$ In the USA, tuberculosis is a reportable disease, and therapy is typically supervised by the community health department. ${ }^{31}$ Contacts of persons with infectious tuberculosis should be screened with tuberculosis skin testing or interferon gamma release assay at the time of discovery and 8-12 weeks later. ${ }^{32}$ Those with positive tests should be treated for latent tuberculosis. ${ }^{26}$ Young children are at increased risk of progression to active disease, and are often given prophylactic isoniazid in consultation with an infectious disease specialist. ${ }^{33}$

Contributors $\mathrm{BB}, \mathrm{JK}, \mathrm{JP}, \mathrm{SD}, \mathrm{LP}$ and SK: Conception and design, acquisition of data or analysis and interpretation of data. BB, JK, JP, SD, LP and SK: Drafting the article or revising it critically for important intellectual content. BB, JK, JP, SD, LP and SK: Final approval of the version published. BB, JK, JP, SD, LP and SK: Agreement to be accountable for the article and to ensure that all questions regarding the accuracy or integrity of the article are investigated and resolved. BB, JK, JP, SD, LP and SK: Agree with revisions and resubmission.

Funding The authors have not declared a specific grant for this research from any funding agency in the public, commercial or not-for-profit sectors.

Competing interests None declared.

Patient consent for publication Obtained.

Provenance and peer review Not commissioned; externally peer-reviewed.

Open access This is an open access article distributed in accordance with the Creative Commons Attribution Non Commercial (CC BY-NC 4.0) license, which permits others to distribute, remix, adapt, build upon this work non-commercially, and license their derivative works on different terms, provided the original work is properly cited and the use is non-commercial. See: http://creativecommons.org/ licenses/by-nc/4.0/.

\section{REFERENCES}

1 Odev K, Guler I, Altinok T, et al. Cystic and cavitary lung lesions in children: radiologic findings with pathologic correlation. J Clin Imaging Sci 2013;3:60.

2 Kim NR, Han J. Pathologic review of cystic and cavitary lung diseases. Korean J Pathol 2012;46:407-14.

3 Sodhi KS, Bhalla AS, Mahomed N, et al. Imaging of thoracic tuberculosis in children: current and future directions. Pediatr Radiol 2017:47:1260-8.

4 Solovan C, Chiticariu E. Psoriasis, anti-tumor necrosis factor therapy, and tuberculosis: report of three challenging cases and literature review. Infect Dis Ther 2013;2:59-73.

5 Salvana EMT, Salata RA. Infectious complications associated with monoclonal antibodies and related small molecules. Clin Microbiol Rev 2009;22:274-90.

6 Pednekar M, Chandra AB, Chandra PA. Disseminated tuberculosis secondary to adalimumab. Am J Ther 2012;19:e139-40.

7 Boushab BM, Basco LK. Miliary tuberculosis and acute respiratory distress syndrome. J Clin Tuberc Other Mycobact Dis 2019;16:100113.
8 Sánchez-Códez MI, Lubián-Gutiérrez M, Fernández-Bravo C, et al. Pediatric miliary tuberculosis presenting with stroke: contribution to the paper "Tuberculosis of the central nervous system in children". Childs Nerv Syst 2019;35:1273-5.

9 Nahid P, Dorman SE, Alipanah N, et al. Official American thoracic Society/Centers for disease control and Prevention/Infectious diseases Society of America clinical practice guidelines: treatment of drug-susceptible tuberculosis. Clin Infect Dis 2016;63:e147-95.

10 Rock RB, Olin M, Baker CA, et al. Central nervous system tuberculosis: pathogenesis and clinical aspects. Clin Microbiol Rev 2008;21:243-61.

11 CDC. Reported tuberculosis in the United States. Available: https://www.cdc.gov/tb/ statistics/reports/2016/default.htm [Accessed 10 Jun 2018].

12 Newton SM, Brent AJ, Anderson S, et al. Paediatric tuberculosis. Lancet Infect Dis 2008;8:498-510

13 Marais BJ, Gie RP, Schaaf HS, et al. Childhood pulmonary tuberculosis: old wisdom and new challenges. Am J Respir Crit Care Med 2006;173:1078-90.

14 Perez-Velez CM, Marais BJ. Tuberculosis in children. N Engl J Med 2012;367:348-61.

15 Cruz AT, Starke JR. Clinical manifestations of tuberculosis in children. Paediatr Respir Rev 2007;8:107-17.

16 Kaba Özge, Kara M, Odacilar CA, et al. Evaluation of cases of pediatric extrapulmonary tuberculosis: a single center experience. Turk Pediatri Ars 2019:54:86-92.

17 Pelayo J, Ruddiman K. Miliary tuberculosis. N Eng/ J Med 2020;383:e78.

18 Kritsaneepaiboon S, Andres MM, Tatco VR, et al. Extrapulmonary involvement in pediatric tuberculosis. Pediatr Radiol 2017:47:1249-59.

19 Wakamatsu K, Nagata N, Kumazoe H, et al. Prognostic factors in patients with miliary tuberculosis. J Clin Tuberc Other Mycobact Dis 2018;12:66-72.

20 Dixon WG, Hyrich KL, Watson KD, et al. Drug-Specific risk of tuberculosis in patients with rheumatoid arthritis treated with anti-TNF therapy: results from the British Society for rheumatology biologics register (BSRBR). Ann Rheum Dis 2010;69:522-8.

21 Keane J, Gershon S, Wise RP, et al. Tuberculosis associated with infliximab, a tumor necrosis factor alpha-neutralizing agent. N Eng/ J Med 2001;345:1098-104.

22 Yaramiş A, Gurkan F, Elevli M, et al. Central nervous system tuberculosis in children: a review of 214 cases. Pediatrics 1998;102:E49.

23 Andronikou S, Smith B, Hatherhill M, et al. Definitive neuroradiological diagnostic features of tuberculous meningitis in children. Pediatr Radiol 2004;34:876-85.

24 Lam KY, Lo CY. A critical examination of adrenal tuberculosis and a 28-year autopsy experience of active tuberculosis. Clin Endocrinol 2001:54:633-9.

25 Lewinsohn DM, Leonard MK, LoBue PA, et al. Official American thoracic Society/ Infectious diseases Society of America/Centers for disease control and prevention clinical practice guidelines: diagnosis of tuberculosis in adults and children. Clin Infect Dis 2017:64:e1-33.

26 CDC. Treatment regimens for latent TB infection (LTBI). Available: https://www.cdc. gov/tb/topic/treatment/ltbi.htm [Accessed 14 Jun 2018].

27 Ruslami R, Ganiem AR, Dian S, et al. Intensified regimen containing rifampicin and moxifloxacin for tuberculous meningitis: an open-label, randomised controlled phase 2 trial. Lancet Infect Dis 2013:13:27-35.

28 Heemskerk AD, Bang ND, Mai NTH, et al. Intensified antituberculosis therapy in adults with tuberculous meningitis. N Engl J Med 2016;374:124-34.

29 Boeree MJ, Heinrich N, Aarnoutse R, et al. High-Dose rifampicin, moxifloxacin, and SQ109 for treating tuberculosis: a multi-arm, multi-stage randomised controlled trial. Lancet Infect Dis 2017;17:39-49.

30 Lanzafame M, Vento S. Tuberculosis-immune reconstitution inflammatory syndrome. J Clin Tuberc Other Mycobact Dis 2016;3:6-9.

31 Marais BJ, Gie RP, Hesseling AC, et al. A refined symptom-based approach to diagnose pulmonary tuberculosis in children. Pediatrics 2006;118:e1350-9.

32 CDC. Guidelines for the investigation of contacts of persons with infectious tuberculosis. Available: https://www.cdc.gov/mmwr/preview/mmwrhtm//rr5415a1.htm [Accessed 14 Jun 2018].

33 CDC. TB in children in the United States. Available: https://www.cdc.gov/tb/topic/ populations/tbinchildren/default.htm [Accessed 14 Jun 2018].

Copyright 2021 BMJ Publishing Group. All rights reserved. For permission to reuse any of this content visit

https://www.bmj.com/company/products-services/rights-and-licensing/permissions/

BMJ Case Report Fellows may re-use this article for personal use and teaching without any further permission.

Become a Fellow of BMJ Case Reports today and you can:

- Submit as many cases as you like

- Enjoy fast sympathetic peer review and rapid publication of accepted articles

- Access all the published articles

- Re-use any of the published material for personal use and teaching without further permission

Customer Service

If you have any further queries about your subscription, please contact our customer services team on +44 (0) 2071111105 or via email at support@bmj.com.

Visit casereports.bmj.com for more articles like this and to become a Fellow 